the much slower change induced in Factin, and from the apparent ability of myosin to displace cytochalasin from actin, that there is direct competition for actin between the two.

The extent of the diminution of the $F$-actin and the actomyosin viscosity under the influence of cytochalasin B is clearly not compatible with complete depolymerization. Either the filaments are shortened or their geometry is drastically changed, in such a way as to increase their cross-section. This is where Forer et al. enter the argument. Their work is based entirely on electron microscopy, and their pictures of actin filaments carrying arrowheads of heavy meromyosin, prepared in the presence and absence of cytochalasin B, are assuredly identical, at least to the innocent eye and the unbiased mind. They checked also that the release (or exchange) of the myosin from the filaments was not inhibited by the cytochalasin, by demonstrating the disappearence of the arrowheads after addition of ATP. Again actin filaments prepared from G-actin solutions containing cytochalasin presented an entirely normal appearance. Forer et al. conclude that cytochalasin B and actin (or actomyosin) do not interact in any interesting way.

Now it is, of course, true that the two studies do not measure directly comparable parameters; indeed the failure of chemical and structural measurements to make contact is no new experience, protein chemists and electron microscopists having often enough glared at each other eyeball to eyeball to no great effect. A reconciliation might in the present case result from electron microscopic measurements of filament lengths. If the effect of the cytochalasin is to chop down the filaments to a defined limit, this would raise some interesting new issues. A small distortion at the inter-strand contact sites could lead to a change in the helix repeat of the actin filaments with a consequent change in length, or if another protein were present an explanation in terms of a vernier effect on the limiting length could be invoked. Then again, one or other of the reported findings might just be wrong.

\section{MAMMARY CARCINOMA \\ Domes of Replication}

from our Cell Biology Correspondent

Mouse mammary tumour viruses have of late caught the limelight not because of any great leap forward in understanding of their biology but because a particle which is structurally and biochemically similar has been detected in some human milks. It is hard to resist the suggestion that these human milk particles are the human counterparts of the murine viruses and, by analogy with the murine situation, that they may have an aetiological role in human breast cancer. And because one of the chief justifications of research into lower mammalian RNA tumour viruses is the belief that there exist similar human viruses yet to be discovered, the detection of human milk virus has strengthened the conviction of the converted and won a few new converts into the bargain.

As luck would have it, however, mouse mammary tumour viruses are far more difficult to experiment with than the murine sarcoma and leukaemia viruses, not least because nobody has yet succeeded in infecting and transforming cultivated cells with mammary tumour virus. Inevitably, therefore, assays of this virus involve slow, costly experiments with mice and the extensive use of electron microscopy, although new reverse transcriptase assays are promising. The search for cultivated cells which support the replication of exogenous mammary tumour virus to yield infectious progeny and which are transformed by the virus continues, but the recent work of McGrath, Nandi and Young (J. Virology, 9, 367; 1972) raises the possibility that the search may inevitably be fruitless.

McGrath, Nandi and Young have placed in culture acinar tissue of primary mouse mammary tumours, which were known to be producing mammary tumour virus, mammary epithelial cells from pregnant BALB/ cfC $3 \mathrm{H}$ mice, which although not bearing tumours were infected by mouse mammary tumour virus, and, finally, mammary epithelium from uninfected healthy mice. When insulin and cortisol are added to these primary cultures the cells organize into threedimensional structures, called domes, which appear to be in vitro analogues of mammary gland acini. The domes, for example, produce abundant casein, and their cells, if from animals infected with mammary tumour virus, support the replication of the virus in culture. By contrast, under a similar culture regi-

\title{
Enhanced Agglutination of RNA Transformants
}

IT is now generally agreed that cells transformed by DNA tumour viruses and chemical carcinogens are more readily agglutinated by the lectins wheat germ agglutinin (WGA) and concanavalin A (Con A) than are the parental untransformed cells. The molecular basis of this is, however, obscure and is currently vigorously disputed.

Apparently, transformation by these agents results in a change either in distribution or number, or both, of the surface glycoproteins to which lectins bind. Of course, if this change in the cell surface, which is detected by agglutinins, has any general physiological significance, one might expect it to be common to all transformed cells irrespective of the nature of the transforming agent, and in Nature New Biology next Wednesday (May 3) Burger and Martin report experiments which, contrary to those of Moore and Temin (Nature, 231, 117; 1971), indicate that that is indeed the case.

Last year Moore and Temin reported that, unlike rodent fibroblasts transformed by polyoma virus or SV40, chick and rodent fibroblasts transformed by several RNA tumour viruses are no more susceptible to agglutination by WGA and Con A than are the corresponding untransformed cells. This disturbing result seemed, on the face of things, to cast grave doubts on the idea that the surface change detected by lectins is causally related to the changed pattern of growth of transformed cells. It occurred to Burger and Martin, however, that the increased amounts of mucopolysaccharides, in particular hyaluronic acid, which accumulate on the surfaces of cells transformed by Rous sarcoma virus (RSV) might interfere with the binding of lectins and agglutination.

Burger and Martin therefore exposed cells to hyaluronidase to strip any masking hyaluronic acid and then measured the agglutinability of untransformed chick fibroblasts and cells transformed by the temperature sensitive mutant of RSV isolated by Martin. This mutant has a temperature sensitive lesion in a gene, the expression of which is required to maintain a cell in the transformed state. They found that after treatment with hyaluronidase untransformed cells remain relatively unsusceptible to agglutination. Cells transformed by the mutant and grown at its permissive temperature are, however, rendered much more susceptible to agglutination by hyaluronidase digestion, but identical cells at the non-permissive temperature are not readily agglutinated even after exposure to the enzyme.

In other words, transformation of fibroblasts by RSV, and by implication other RNA tumour viruses, leads to at least some of the changes to the cell surface caused by transformation by DNA tumour viruses. Furthermore, with both RNA and DNA tumour viruses the maintenance of this changed cell surface depends, however indirectly, on the continuous expression of at least one viral gene. Essentially similar conclusions have, of course, been reached by Capeller and Doljanski (Nature New Biology, 235, 184 ; 1972). 